\title{
EMPLEABILIDAD DE LOS DOCENTES QUE APLICAN LA ESTRATEGIA METACAFÉ LECTOR EN LA UNIVERSIDAD RICARDO PALMA
}

\author{
Úrsula ROMANI MIRANDA \\ Universidad Ricardo Palma \\ ursula.romani@urp.edu.pe \\ Jorge RIVERA MUÑOZ \\ Universidad Nacional Mayor de San Marcos \\ jriveram@unmsm.edu.pe
}

\section{RESUMEN}

El presente artículo de revisión bibliográfica consiste, en observar y describir la empleabilidad de los docentes que aplican la estrategia Metacafé Lector, en la Universidad Ricardo Palma. La empleabilidad presenta diferentes indicadores como la actualización del profesional quien debe estar a la vanguardia de los cambios y evolución de las nuevas generaciones $y$, quien en su rol de dicho proceso, debe buscar solucionar las debilidades académicas. En realidad, se trata, de que los estudiantes se sientan en un mejor clima de aprendizaje - enseñanza cumpliendo así, con las competencias establecidas en los sílabos. Y, todo esto, se evidencia entre los colegas y autoridades de la universidad. El objetivo general de la investigación es interpretar la empleabilidad de los docentes que aplican la estrategia en mención.

\section{Palabras claves}

Empleabilidad. Clase invertida. Metacafé Lector. Tecnología.

\section{EMPLOYABILITY OF TEACHERS WHO APPLY THE METACAFÉ READER STRATEGY AT THE RICARDO PALMA UNIVERSITY}

\section{ABSTRAC}

This bibliographic review article consists of observing and describing the employability of teachers who apply the Metacafé Lector strategy, at Ricardo Palma University. Employability presents different indicators such as the update of the professional who must be at the forefront of the changes and evolution of the new generations and, who in his role of said process, must seek to solve academic weaknesses. Actually, it is about students sitting in a better learning environment - teaching thus complying with the competencies established in the syllables. And, all this is evident among the colleagues and authorities of the university. The general objective of the research is to interpret the employability of the teachers who apply the strategy in question.

\section{KEY WORDS}

Employability. Inverted Class. Metacafé Reader. Technology. 


\section{INTRODUCCIÓN}

os docentes universitarios a diferencia de otros profesionales en su perfil deben evidenciar algunas competencias como las informáticas que les ayuden a desarrollar el proceso de aprendizaje - enseñanza. Cuando se poseen pueden sentirse dentro del nuevo enfoque educativo. En este sentido, se hace medular cuán empleable puede ser un trabajador en una organización, si está preparado para ser parte del cambio y el desarrollo de la sociedad o no. García y Aguilar (2011)"la empleabilidad entendida como la capacidad de las personas para encontrar trabajo y ser competitivo en el mercado laboral [...] consistiría en desarrollar la capacidad para encontrar un trabajo de calidad que permita a las personas crecer profesionalmente, incluso percibiendo los cambios laborales como oportunidades profesionales de mejora, es decir, aquellos elementos competitivos que añaden valor a un profesional y lo diferencian del resto a la hora de optar a un puesto de trabajo" En el siguiente artículo, se desarrollará el marco teórico sobre la empleabilidad, la estrategia del Metacafé Lector, las experiencias de la estrategia y la conclusión.

\section{EMPLEABILIDAD}

OIT (2004), la empleabilidad quedaría referida a las competencias y cualificaciones transferibles, reforzando así la capacidad de las personas para aprovechar las oportunidades de educación y formación. La finalidad estriba, por tanto, en alcanzar objetivos tales como encontrar y conservar un trabajo óptimo; progresar en la empresa o cambiar de empleo y, adaptarse a la evolución de la tecnología y las distintas condiciones del mercado de trabajo. Así, la empleabilidad queda asociada a un concepto multidimensional y complejo, en el que el aprendizaje y el desarrollo de competencias se interpreta como una continuidad (Harvey, 2001).

La empleabilidad no puede considerarse como una garantía plena desempleo real, pero sí puede incrementar las posibilidades para ello (Nilsson y Ekberg, 2013).

Gamboa et al. (2012) define la empleabilidad como "la percepción del individuo sobre las oportunidades que tiene de conseguir un empleo de su preferencia o de mejorar el que posee, teniendo en cuenta que dichas oportunidades van a depender de sus características y conductas y de los factores contextuales que le rodean".

Temple (2015) define "la empleabilidad es la capacidad o facultad que tiene una persona de mantener o mejorar su empleo actual o lograr uno nuevo, de igual o mejor nivel de satisfacción profesional/ personal que el actual, en un tiempo determinado" (p.31).

Rivera y Romaní (2016) [...] adquirir las competencias informáticas como docentes es muy útil ya que los estudiantes corresponden a una generación digital y ellos se sentirán más familiarizados y motivados con el uso de las TAC. Solo así podremos aplicar estrategias como la clase invertida que consiste en realizar sesiones virtuales previas antes de realizar las presenciales. 
Rivera y Romaní (2016), se evidencia que dentro de las competencias genéricas se busca desarrollar las competencias tecnológicas, pues en esta sociedad del conocimiento es necesario en primer momento buscar y tener un manejo de la información y para ello el conocimiento de las TIC (Tecnologías de la información y comunicación), TAC (Tecnologías del aprendizaje y el conocimiento) y TEP (Tecnología del empoderamiento y participación). Las mismas que van acorde con las corrientes de la conectividad en la educación, ya que las competencias permiten responder de manera más concretas a las exigencias mundiales. Tal como lo afirma Lozano (2011) "las TAC van más allá de aprender meramente a usar las TIC y apuestan por explorar estas herramientas tecnológicas al servicio del aprendizaje y de la adquisición de conocimiento". Por ello, se debe buscar que las técnicas e instrumentos de desempeño docente se puedan aplicar en las TAC.

En cuanto a la empleabilidad del docente universitario consiste en responder a un perfil, el mismo que debe presentar competencias digitales, con la finalidad de alcanzar el Objetivo de Desarrollo Sostenible n. 4 "Educación de Calidad", la cual precisa a través de Meta 4.4 Aumentar sustancialmente el número de jóvenes y adultos que tienen las competencias necesarias, en particular técnicas y profesionales, para acceder al empleo, el trabajo docente y el emprendimiento: 4.4.1 Proporción de jóvenes y adultos que han adquirido competencias de tecnologías de la información y comunicación (TIC), por tipo de conocimiento técnico y el 4.4.2 Porcentaje de jóvenes y adultos que han alcanzado al menos un nivel mínimo de competencia en alfabetización digital. Dado a que de esa manera podrán responder a los actuales paradigmas educativos como es la conectividad.

Por otro lado, los estándares del Modelo de Acreditación para Programas de Estudios de Educación Superior Universitaria establecidos por el Sistema Nacional de Evaluación, Acreditación y Certificación de Calidad de la Educativa (2016), a través de la dimensión 2: FORMACIÓN INTEGRAL Factor 4. PROCESO ENSEÑANZA APRENDIZAJE El programa de estudios gestiona el documento curricular, incluyendo un plan de estudios flexible que asegure una formación integral y el logro de las competencias a lo largo de la formación. El proceso de enseñanza aprendizaje está articulado con la investigación, desarrollo tecnológico, innovación y responsabilidad social, así como fortalecido por el intercambio de experiencias nacionales e internacionales. (p. 16). Observamos que la tecnología debería estar presente en el proceso de enseñanza - aprendizaje y para eso el docente debería estar capacitado en las competencias digitales. Asimismo, Unesco (2008) en Estándares de Competencia en TIC para docentes dice refiriéndose al papel del docente en el enfoque relativo a la generación del conocimiento que la función de los docentes consiste en modelar abiertamente procesos de aprendizaje, estructurar situaciones en las que los estudiantes apliquen sus competencias cognitivas y ayudar a los estudiantes a adquirirlas. (Granados, 2015, p. 146).

En este marco es que surge la estrategia del Metacafé Lector donde se emplean recursos tecnológicos y en un nuevo modelo pedagógico que está relacionado con la conectividad que es la clase invertida, sin perder la esencia de un educador que es desarrollar valores, donde el docente se acerca más al estudiante en un enfoque por competencias. 


\section{La estrategia Metacafé Lector}

Esta estrategia de metacomunicación se sustenta en el uso del modelo de la clase invertida y el buen clima en el aula; se realiza la interacción, después de la comprensión de los textos leídos. Dado que los estudiantes ya vienen a clases con aprendizajes previos de lo que se realizará en clases, asimismo, se habrá organizado para desarrollar y conversar sobre preguntas presentadas en cuestionarios como Kahoot y Socrative mientras preparan y consumen café.

\section{Fases de la estrategia Metacafé Lector}

\section{Primera fase}

Se organiza el aula para la actividad del Metacafé lector, aquí se desarrollan las relaciones interpersonales (sociales), la misma que es la base de un buen clima para desarrollar el proceso de aprendizaje - enseñanza, ya que deben practicar el respeto a la delegación de funciones y cumplimiento, asimismo, realizar escucha activa e interactuar con una comunicación asertiva, efectiva y eficaz, la misma que se alcanzará con la metacomunicación. En esta fase, los estudiantes designan quienes traerán los utensilios (tazas, cucharas y hervidor) para llevar a cabo la preparación de un café en el aula. Además, cada uno debe procurar traer objetos que se puedan reciclar. Por último, el delegado elabora un cronograma para hacer el recordatorio de las actividades y compromisos asumidos.

\section{Segunda fase}

Los estudiantes ingresan a la plataforma virtual (FRONTER, MOODLE, CHAMILO, $B L A C K B O A R D$ ) o en el Blog del curso donde se ha credo un espacio ergonómico con los recursos tecnológicos como son las presentaciones efectivas como Pow toon, Emaze, Prezi, asimismo, los estudiantes encontrarán material bibliográfico sobre los temas que se abordarán en el aula, los mismos que están relacionados a la comprensión de textos como obras "Tradiciones Peruanas", artículos científicos de temas de sus carreras y sobre problemas ambientales, equidad e igualdad de derechos de género, el feminicidio, entre otros. finalmente, elaboran sus resúmenes en fichas. En otros términos, se emplea el modelo de la clase invertida o flipped classroom.

De acuerdo a Olaizola (2014): "El flipped classroom o clase invertida es un modelo pedagógico que se basa en la inversión de la estructura tradicional de la clase presencial expositiva a través del empleo de las TIC."

En consecuencia, la retroalimentación y ajuste de los temas leídos se realiza en el aula. Debido a que nos aseguramos que existe un conocimiento previo sobre lo que se abordará en clases y profundizará a través de estrategias que respondan a las competencias de cada asignatura o curso.

\section{Tercera fase}

En esta última fase, los estudiantes asisten a clases con saberes previos; dado a que ya saben de qué se trata el tema del conversatorio que se realizará en el aula. También se realizan preguntas en los tres niveles de comprensión de textos: literal, 
inferencial y crítico a través de la aplicación Socrative o Kahoot. Estos se realizan de manera gradual. Previamente, se ordena el aula donde las carpetas forman un círculo y de esta manera se podrán visualizar a todos los estudiantes y mantener una comunicación horizontal o formar equipos de trabajo para resolver las preguntas del aplicativo a través de sus celulares.

Luego los mismos estudiantes colocan una mesa para preparar el café en el aula. Finalmente, se sirve el café y el docente genera las preguntas en nivel crítico. En esta fase, se observa alegría y convivencia entre los estudiantes donde expresan la metacomunicación, pues los encontrarse en un clima amical donde a través del diálogo comparten sus puntos de vistas al responder las preguntas del nivel crítico; dado que se sienten en libertad en exponer sus posturas y sustentarlas. Es en esta última fase, donde se evidencian los valores de tolerancia, asertividad y escucha activa.

Es necesario resaltar que la concepción cognitiva del aprendizaje postula que el aprendizaje significativo ocurre cuando la persona interactúa con su entorno y de esta manera construye sus representaciones personales, por lo que, es necesario que realice juicios de valor que le permiten tomar decisiones en base a ciertos parámetros de referencia.

Como la estrategia del Metacafé Lector es digital, no solo por el uso de la plataforma y presentaciones efectivas, sino más aún por el uso de la gamificación (Kahoot y Socrative) tiene un rol importante; puesto que une a las generaciones de estudiantes con los docentes; por ende, nos hace más empleables en nuestras aulas. Tal como menciona Álvarez (2019), sostiene que la gamificación al ser incorporada en el aprendizaje debe considerar:

> Identificar la característica del estudiante, para emplear en clase la herramienta más adecuada, concordante con las habilidades del aprendiz y detectar el nivel de dificultad de la actividad con criterios motivacionales.

> Establecer los propósitos de aprendizaje, que deben ser específicos y permitirá valorar donde es posible insertar la gamificación generando resultados favorables.

> Producción de contenido y actividades para la gamificación, que debe ser interactivo, atractivo y pertinente con elementos multimediales, es decir, diseñados para posibilitar varias oportunidades que garanticen llegar al propósito de aprendizaje, es decir un reto alcanzable, superable, mejorable en sus habilidades en la perspectiva de que el estudiante construya sus propias estrategias.

> Adhesión de elementos de los juegos e inclusión de tareas que los estudiantes puedan realizar. Toda actividad que realicen debe estar ligada a los objetivos y logros de aprendizaje.

\section{Experiencia en aulas universitarias}

Desde el año 2014 hasta la fecha se ha aplicado la estrategia del Metacafé Lector entre los estudiantes universitarios de la Universidad Científica del Sur. Se ha 
evidenciado motivación e integridad entre los estudiantes y docentes. Donde se observa que es posible aprender y desarrollar las competencias de la comprensión y expresión oral dentro de un buen clima de aula. Esto, se ha visto reflejado en el logro de las competencias y mejoras en muchos de los casos. Se observó que tuvo buenos resultados en el curso de Lengua y Comunicación (las notas subieron dentro de esta competencia, así también la asistencia fue más permanente en los estudiantes en las clases); por ello, se quiso hacer una resonancia en otros cursos como en el de Filosofía en el semestre 2015-II. Dado a que los jóvenes comentaban la actividad y que les agradaba compartir lo leído y sentirse entre amigos.

En la estrategia del Metacafé Lector se evidenció la participación activa de los estudiantes e incluso de organizarse internamente para llevar a cabo la sesión de aprendizaje sobre los niveles de comprensión en una población de 640 estudiantes.

Los docentes generaron preguntas en los tres niveles de comprensión de textos que permitan la participación oral y la discusión en el aula. Asimismo, el registro de cada participación, solo así se evidenciará la efectividad de la estrategia. Además, debo acotar que este año se ha empleado textos según las carreras que desarrollan.

En el año 2018, se aplicó a ocho grupos de estudiantes con un número de 240 estudiantes de pregrado del programa PEB de la Universidad Ricardo Palma. Los estudiantes mostraron notablemente mejorar sus niveles de comprensión lectora, así como en su redacción. Asimismo, se realizó capacitaciones a los docentes del curso de Taller de Comunicación Oral- Escrita I.

\section{Beneficios de aplicar la estrategia}

$>$ Promover la comprensión de los textos académicos.

> Fortalecer los valores inherentes al asertividad, empatía, escucha activa, respeto, responsabilidad y tolerancia entre todos.

> Generar un buen clima de aprendizaje, es decir están metacomunicados, lo cual facilita una comunicación interactiva.

$>$ Promover la creatividad de los docentes y dar respuestas que se ajusten a las necesidades de cada generación estudiantil.

> Al emplearse el flipped classroom, es innecesaria la impresión de los materiales del curso.

$>$ Al usarse los recursos tecnológicos como el Socrative o el Kahoot, es innecesario hacer evaluaciones escritas.

Al lograr una comprensión en el nivel crítico, los estudiantes pueden generar acciones positivas y de consciencia en la sociedad sobre los problemas sociales como: los ambientales, los de equidad e igualdad de derechos, la no violencia hacia la mujer y el feminicidio, entre otros. 


\section{CONCLUSIÓN}

Al ser el docente una persona actualizada y aplicar las tecnologías se convierte en una persona empleable, pues cumple con efectividad en el proceso de enseñanzaaprendizaje, pues una de las competencias profesionales que deben cumplir los docentes en el nivel universitario son las Habilidades en el uso de las tecnologías de la información y de la comunicación. De la misma forma, el docente siente que está creciendo profesionalmente, pues aplica estrategias que ayudan a alcanzar las competencias comunicativas empleando la tecnología de vanguardia.

\section{REFERENCIAS}

Conde, J. (2017). La mediación de las TIC en la creación de ambientes de aprendizaje y el logro de competencias digitales. (tesis doctoral). Universidad de Sevilla, España. Recuperado de https://dialnet.unirioja.es/servlet/tesis?codigo $=6851$

Gamboa, J. (2013). La Empleabilidad de los Jóvenes como Facilitadora de la Obtención de Empleos de Calidad. (tesis Doctoral). Universidad de Valencia, España. Recuperado de https://dialnet.unirioja.es/servlet/tesis?codigo $=65036$

García, F. (2015). Empleabilidad de Pedagogo(a) a través del análisis interpretativo de experiencias profesionales. (tesis doctoral). Universidad de Málaga, España. Recuperado de https://dialnet.unirioja.es/servlet/tesis?codigo=78526

Gonzales R., Juan M. (2010). Comentario crítico de texto. Trivium. Sevilla. Recuperado de http://www.lenguayliteratura.net/index.php?option=com_content\&view

International Labuor Organization (2014). Mejorar la empleabilidad de los jóvenes: la importancia de las competencias clave. Competencias para el empleo orientaciones de la política.1-7. Recuperado de https://www.lo.org/wcmsp5/ groups/public/---ed_emp/---.../wcms_371815.pdf

Rivera, J. (2014). Impacto de las tecnologías de información y comunicación en los procesos de enseñanza-aprendizaje. Investigación Educativa, [S.I.], 15 (27), p. 127 - 138. ISSN 1728-5852. Recuperado de http://revistasinvestigacion.unmsm. edu. pe/index.php/educa/article/view/5192

Romaní, U. y Rivera, J. (2017). Estrategias en la educación superior por competencias. Revista Scientia, 13(18), pp. 285-294 CIUP.

Temple, I. (2015). Usted S.A. Empleabilidad y marketing personal. Lima, Perú: Planeta

Vásquez, M. (2016). Evaluación de las Competencias Básicas en TIC en docentes de educación superior en México. (tesis doctoral). Universidad Nacional de Educación a Distancia, España. Recuperado de https://dialnet.unirioja.es/ servlet/tesis?codigo $=64124$ 